\title{
Research on Teaching Materials Management of Track and Field Web Course in College of Physical Education
}

\author{
http://dx.doi.org/10.3991/ijet.v11i06.5460 \\ Weiliang Lin \\ Guangzhou University, Guangzhou, China
}

\begin{abstract}
The purpose of this research is to analyze the teaching material management and its effect on the web course, using Track and Field (T\&F) web course in Guangzhou University as an example. Methods: the research methods include literature, expert interview, questionnaire survey, mathematical statistics, system settings, software editing etc. Results: My resources have abundant teaching materials in a variety types which gives access to resource re-editing and transmission. The visual effect of the teaching content and structure is excellent which helps the sustainable update management of the resources. The syllabus is specific; resources collection and modular management are effective. Teaching resources include materials combining theory and practice with many effective management forms. Test materials can help effectively designed assignments and test papers for teaching tasks. Conclusion: Teaching material module has the essential characteristics, content-rich system, complementary resources and resource sharing; it is convenient for the implementation of the teaching tasks and the integration of teaching materials. This study applies to physical education majors and to the multi-functional setting management of teaching materials in $T \& F$ web course.
\end{abstract}

Index Terms - college, physical education, track and field web course, teaching materials, management

\section{INTRODUCTION}

Teaching resources management model is reflected in computer, network videos, domestic and international videos and vocational resources. In the current industrial culture, vocational resource management includes teaching material classification and cultural environment support which is extracted from practice, required by the social demand of the profession $[2,4,9]$. In the management of network materials, the proposal of a new theory or practical problems is conducive to the construction of network platform and the enhancement of the innovative collection of theoretical and actual cases $[1,3,20]$. Class management, on-line and off-line teaching management, online examinations, assignments and marking management proposed in the Web Course Management System. Basic Computer Courses will be effective and be a good way to improve the implementation, evaluation and communication of teaching tasks $[7,10,14]$. In the open network course video resource management, web course resources at home and abroad are very different and due to the lack of systematic construction of professional information and network technology, the web course aboard are more advantageous in the comparative research $[5,15,18]$. In the Net-Course function module man- agement, the establishment and management of video communication area for students and teachers are of high reference value $[11,13,16]$.

So far, in construction of web courses, more emphasis are given on collaborative teaching activities, team innovation spirit, idea exchanges and answering evaluation system $[6,12,17,19]$. My question answering module includes problem upload, teacher answering, answer upload between teachers and students, and its technical staff. Administrators play a guiding role and keep continuous operation of the module resource management $[8,25,26]$.

Currently, the research on the relevant theoretical system of T\&F web course is mainly reflected in the following respects: the design mode of web course of $T \& F$ in physical education [21] the basic attributes [22], course content [23], course parameters, class and members. The settings of T\&F assignment database include the settings of test library and test management, teacher-student forum management. T\&F course introduction, $T \& F$ reference materials, which have been in-depth studied and applied in practice [24]. However, there is a lack of literature for management of web course teaching resources of $T \& F$. So, the in-depth and comprehensive study on web course teaching resources management models, such as my resources, teaching structure, teaching syllabus, textbook, tests, resource links, which will help improve the material classification, updating, complementation, resource section management effect of T\&F web course. These have some practical significance.

\section{Study OBjects And Methodology}

\section{A. Study objects}

Take the web course of T\&F in Guangzhou University as a case study and the teaching materials management of T\&F web course in College of Physical Education.

\section{B. Methods}

Literature study: Search for the cases of college web course teaching materials management at home and abroad over the years, particularly the references on my resources, teaching structure resource, teaching syllabus materials, textbook resource management in college $\mathrm{T} \& \mathrm{~F}$ web course. Moreover, search in the authoritative journals related to the platform construction of online course's materials library, textbook resource application to lay a basis for this web course's teaching materials management.

Interview: Further collecting comments and suggestions from experts and professors from the network center and 
School of Physical Education in Guangzhou University; analyze the comprehensive construction distributions of material library, textbook resource application, teaching tests resource and resource links management in the web course teaching materials management to make sure the effectiveness of the course's teaching materials management.

Questionnaire survey: On 18 June 2015, 210 sets of questionnaires referring to the T\&F web course had been handed over to 30 experts and 180 students (the number of the questionnaires handed over in each grade is 45 in physical education, among a total of 180 students) with random sampling methods in the network center and in track and field teaching field in Guangzhou University. All questionnaires were collected back, and the collection rate is $100 \%$.

Validity Test of Questionnaires: Delphi Method is adopted in this research and 20 experts were asked to assess the questionnaires in the aspects of "content validity" and "structure validity" to guarantee the validity and reliability of the questionnaires. The result shows that 8 of the experts evaluated that the effect of the questionnaires content is very high, 11 evaluated high and 1 average; 7 of the experts' evaluation is very high to the structure validity, 10 evaluated high and 3 average; none of them evaluated it as low or very low. Generally, the questionnaires have high validity and meet the requirements of the research project.

Reliability Test of Questionnaires: In order to improve the stability of the questionnaires, the accuracy of the assessment should be stable and unanimous. Small sample retest method is adopted in the reliability test of questionnaires. A small number of samples were drawn from the total samples as the objects of the second test which was carried out in 15 days. The test correlation index $r=0.768$ which is high in questionnaire reliability.

The mathematical statistics: SPSS 16.0 statistical software were used to account the 210 sets of questionnaires in order to assess the effects of teaching materials management of its network course from the experts and students of the network center and class teaching in Guangzhou University. It provided empirical evidence for the study.

System Design Method: The method determines the functions of the course's teaching modules and their organic combination according to the learning theories, the teaching requirements and the logical relationship between the content and resources of teaching to improve the actual application effects.

Software programming method: According to the web course designing requirements of specifications of Modern Remote Resource Design Technique was carried out by Ministry of Education in 2000. This platform is developed with software, such as flash, premiere and WCB courseware making tool produced through the cooperation between Guangzhou University and Nanjing Qiaomu Science and Technology Ltd.

\section{TEACHING MATERIAl MANAGEMENT}

\section{A. My Resources Management}

My Resources module includes materials like T\&F syllabus, teaching content and structure, electronic textbooks, practice guidance, video area, exercises and self-tests. My

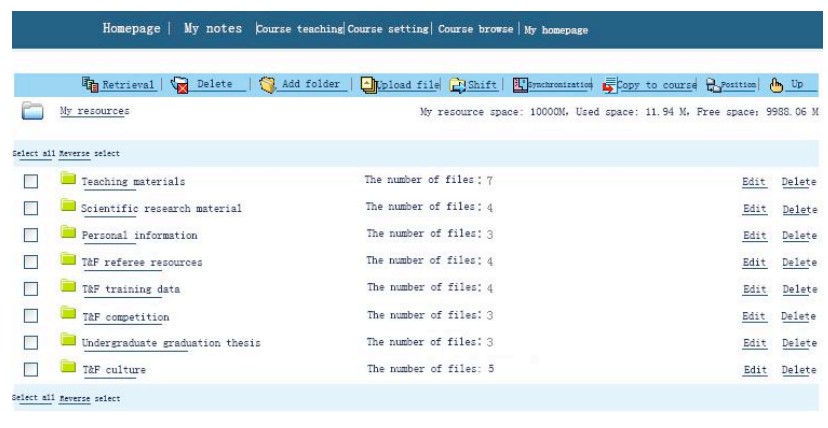

Figure 1. My resources

Resources also include classified materials and undefined materials; timely updating of the resources and the content is diverse in types, materials that would be used recently or in future are predicted. Besides, administrator should search for potential innovative materials and update the teaching resources regularly according to the teaching development of the course. The teaching materials can be transferred, moved and modified for re-use. This section shows the available space, the used space, the free space, folder name and number of files of My Resources. User can also edit file names and delete files (Figure 1).

File name, file size, type of resources and upload time are displayed in the folder; the management features of browse, edit and delete are also provided in My Resources. Each column is with multiple function keys for file management, resource search, delete resource, add folders, upload files, move, resources synchronization, copy to course, positioning and up.

Resource search: materials can be found accurately through setting resource name, resource type, file size, date range and up loader. The number of items displayed in the list can be set $(10,20,30 /$ per page), the features of delete, move and return are provided. Click "browse" to enter the resource information to check its basic property: resource name, up loader user number (1004001), upload time (yyyy-mm-dd, hour-minute-second). Resource type: resource introduction (brief introduction), click "browse" in resource content to view its content, click "close" to exit. Delete resource: select a resource or multiple resources in My Resources, click "delete resource" to delete the resource.

Add folder: firstly, fill in with the folder name; then click "submit" to finish the operation and click "cancel" to return. The folder can be deleted and its name can be edited. The user click on the folder, enter the folder directory, and check the resource space of each folder (the largest available memory: $10000 \mathrm{M}$ ), occupied space and free space.

Upload files: fill in the file's property, upload type (required items: upload physical file- resource name, physical file - browse local "my documents, disk D, disk C" choose documents, resource type --- CSF file, Kejian online tutoring documents, Word files, text files, PPT files, compressed files, FLASH animations, EXCEL files, RM videos, and other documents as required items), Resource description: the content of the document are described with concise language, submit to complete the file upload, cancel to return. Link address, add URL dialog (required), upload type, resource name and the text content (the content can be edited in format, font style, size, background color, document module, browsing, image, video, Excel format, expression and special symbols). 
Resources synchronization: click resource synchronization virtual directory, physical path, choose synchronize personnel, click personnel selection list to set the number of items displayed on each page $(10,20,30$ items), personnel number, name, department name, choose item and click query or query all, and finally click "choose" to complete personnel resources synchronization. Copy to course: choose copy to course structure, tick "track and field two" and directory, click "submit" to complete the operation. Location: the location of the file in the course section. Up: return to the previous page.

\section{B. Teaching Material Management Mode}

Teaching Material Structure Management: The title of course view can be modified through video structure management module. Whether to set the default view or whether to enable the regular interval design or delete the content, click Edit to enter content updates and modifications. The user can add course view and delete items in bulk by ticking them. The number of items displayed per page can be set $(10,20,30,50,75,100)$, view name query or query all the course names can be operated (Figure 2).

The maintenance interface of teaching materials manages the teaching materials and their directory structures. It has the features of add directory, edit directory, locate, move and create similar. The contents added include directories such as files, texts, links, teaching reference, test papers, assignments, new test papers and my resources. Move: select one or more entries to move, click on the "move" button, select the directory to transfer from the page popped out. The numbers $(1,2,3)$ or the items can be adjusted (Figure 2).

Delete in bulk: select one or more entries, click on the "bulk delete" to delete all entries of the subject matter. Up: click on the "up" button to return to the previous page. Locate: select a directory and the directory can be located, the current entry's exact location of which displays a hierarchical directory tree of sub-directories. The user can click the directory name in a directory structure view, then jump to the corresponding directory as the adjustment of the position of the item. Highlight is as follows:

Select the relevant records and the directory to be transferred, click on the directory and enter directly into subdirectories or the folder name. Brief introduction and last modification date will be displayed, then click Edit to edit the content ("delete" is to delete the name of the project and its contents). Add dialog box options are provided in each module: create similar, add directory, add file, add text, add content, add repository, add assignments, add test papers and add new test papers (Figure 2).

Create similar: dendrite form of hierarchical directory indicates the storage form of main directory - subdirectory - directory. Add directory: enter specific content, such as directory name, displayed or not (yes, no). Contents Introduction is to further explain the nature of the directory, press "Submit" button to save the data and return to the list page, click "Back" to give up and return to the list page directly (Figure 2).

In Add File dialog box, fill in with the file title(required), the user can check "sync to my resource"; browsing related options to select files to upload, and choose whether to display items (yes, no); in document description dialog box, fill in with a brief content description; choose a sub-entry of the course "T\&F Two" (such

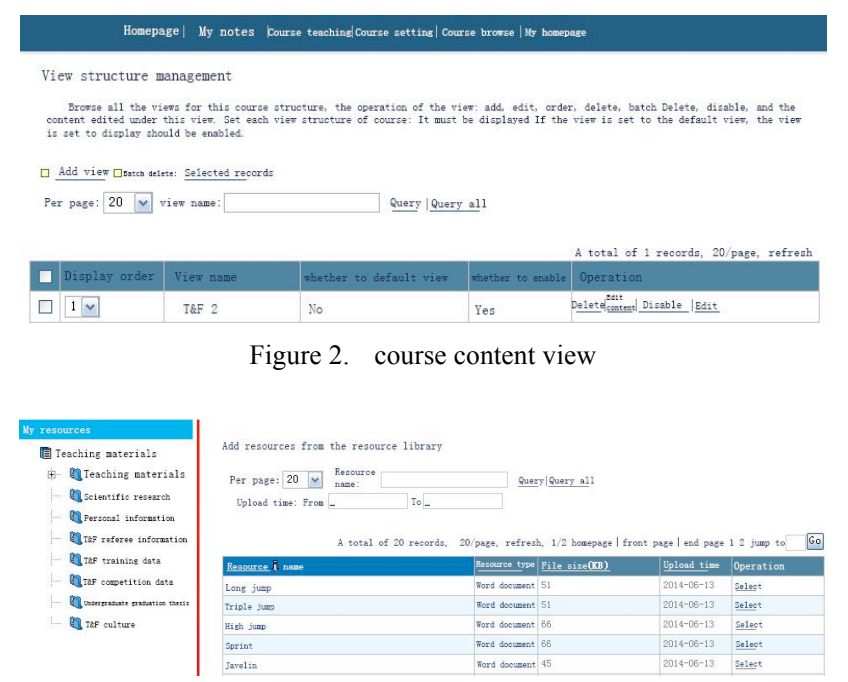

Figure 3. Adding Resources

as teaching methods) in structure dialog box, click "Submit" to save the data and return to the list page, click "Back" to discard data upload and return directly to the list page (Figure 3).

Add text: select "Add Text" in the Add dialog box to fill in the box with the text title (required), "Sync to my resources" can be checked; font art, pictures, videos, web resources, animation, special symbols, background color tone can be adopted for editing of text content to enhance the visual effect of the text. The interface automatically displays the current selection structure, text themes (such as T\&F Two - subdirectory "Teaching Methods") is operating on this view below (Figure 3).

Add content: fill in the repository content, click "Select Resources" in resource name box to select the relevant resource names; the user can also enter the repository through clicking resource directory classification, adjust the number of items displayed per page $(10,20,30)$, resource name, upload period and other conditions to search, or query all the resources. To read the existing resource names, resource types, file size, upload time and other conditions, click on the "Select" under the operation bar to complete adding resources (Figure 3).

Syllabus Material Management: Syllabus materials include teaching methods, teaching calendar and syllabus. Teaching methods reflect in the induction and classification of independent study, self-testing the effect of teaching task, creating methods to collect online course resources, real-time and non-real-time participation in the network teaching activity, course evaluation teaching methods, difference analysis of online teaching methods and practice teaching. These materials improve the teaching effects with graphics, text, video and other multiediting tools.

Teaching calendar: teaching calendars include university calendar and school calendar. The university calendar consists of teaching calendars of every course of the two semesters in the school year. The calendar contains the dates, students and teachers get back to school, school days, public holidays, school events, the end of semesters and vacation time. School calendar is developed based on the university calendar that consists specific plans of the semesters, such as the dates students of physical education get back to school, the quality test period, the project appraisal time, out school internship period, Specialized 
Physical Education course (Sports education, social sports) period, observation period of teaching sessions, practice teaching period, academic conferences dates, thesis defense dates of graduates and dates to leave school.

Syllabus: syllabus includes recent physical education teaching schedule and teaching progress. The T\&F syllabus use the recently updated syllabus like compulsory $\mathrm{T} \& \mathrm{~F}$ courses, elective courses and minor courses as the guide of course teaching. Each course module also comes with the teaching progress of different grades which reflects the teaching content, task, chief instructor and location.

\section{Textbook Resource Management}

Electronic Material: Electronic materials include materials about $\mathrm{T} \& \mathrm{~F}$ referee, $\mathrm{T} \& \mathrm{~F}$ teaching, training, fitness, culture, scientific research, games and competitions. T\&F referee includes the Chinese and English versions of T\&F Refereeing Rules revised by IAAF periodically, new versions of books and illustrated books on the T\&F referee rules. T\&F teaching includes newly published books related to the field of teaching such as teaching methods of throwing, walking and running and biomechanical teaching analysis. $\mathrm{T} \& \mathrm{~F}$ training includes technical training, physical training, strength training, speed training and quality training. $\mathrm{T} \& \mathrm{~F}$ fitness exercise includes fitness methods and means, fitness effective keys etc. T\&F culture contains related historical sources humanistic stories, historical sources of T\&F events, documents and books on $\mathrm{T} \& \mathrm{~F}$ activities and competitions etc. $\mathrm{T} \& \mathrm{~F}$ research is based on the materials of teaching, training, fitness of T\&F. T\&F games include books on game method, organization and content, such as: T\&F Game Method and T\&F Recreational Activities Method.

Practical Guide: Training: the materials are collected from the pre-event training methods used on school students participating in athletics competitions (including national and regional university athletics games, contest, and school sports meetings). The pictures of the training and brief text description can help the construction of students' training systems. At the same time, some issues were analyzed using positive and negative practice cases, such as fallible training methods and proper training methods and means.

Referee: documents and books on new T\&F rules refereeing cases of school teachers and students in T\&F games. It includes the annual school track meetings organized by schools and universities; the form of referee organization in domestic and international championships; the materials reflect the representative features of the cases and the materials are updated regularly. The content is displayed with picture and texts and miniature videos which conform to the cognitive students. The materials are stored in the modules such as "My Resources" and "Practical Guidance". The editing of the materials are mainly done through course material editing software, Leawo video Transcription Software, Adobe Photoshop cc Software, pictures cutting and QQ shots etc. which meet the input conditions of the dialog.

$T \& F$ events: this module includes the results, skills, rules, state of mind, the spirit of cooperation and perseverance presented by the athletes participating in events; optimized technical movements collected from different events, through the process athletes finishing the competition according to the referee rule. It also includes athletic psychological characteristics showed through videos of successful and failed cases with the implementation of the current progress in the task of teaching and is presented as an on-line teaching materials.

Classroom teaching of $T \& F$ : this module includes innovative collections like lesson plans, site equipment preparation, technical terms, standardized demonstration, form of the organized course and its content, the reasonable allocation of exercise load and T\&F events in and out of school. Documents are edited and saved to "My Resources" previously and displayed in the classroom as synchronous materials according to the class progress.

Off campus teaching practice: collection of materials from teaching practice organized by the school, such as teaching-assistance, observation of primary and secondary school teaching, term final teaching practice (skill preparation before practice, teaching, training, team leading and after competition summary). They are edited and saved to "My Resources", and uploaded to the off campus practice module as synchronous materials according to the class progress. These greatly help improving the learning materials before the students' practice.

$\mathrm{T} \& \mathrm{~F}$ research includes research object, research methods, research results and conclusions, collection of subject topic directory, fallible issues, and references for degree thesis topics, popular research topic analysis and bachelor thesis sample reading.

Careers guidance: the materials for college students applying for primary and secondary school physical education positions such as recruitment conditions, candidates quality requirements, evaluation forms, teacher certifications and the employment situation at different times are collected and saved in "My Resources" module as a spare "career guidance" module database. These are uploaded the learning resources according to the teaching needs.

$T \& F$ health knowledge base: the classified materials of bodily injuries caused by T\&F teaching, training, competitions and the treatment of traditional Chinese medicine, and the cases of the combined treatment of traditional Chinese medicine and western medicine are stored in "My Resources" or directly edited as "health knowledge" module.

T\&F Celebrities' module includes Chinese celebrities and foreign celebrities in T\&F and experts on T\&F scientific research. Athletic celebrities are collected according to their achievements in international and domestic events and their spiritual influence on the society. Materials of experts are collected with the consideration of their innovative research results in $\mathrm{T} \& \mathrm{~F}$ training, teaching and sports engineering etc. and their moving deeds as learning materials.

Video Area: Video area includes directories as follows: $\mathrm{T} \& \mathrm{~F}$ training, $\mathrm{T} \& \mathrm{~F}$ referee, events, classroom teaching, off campus teaching practice, $T \& F$ research and career guidance, health knowledge and celebrities etc. Its material contents and practice resources are consistent and they are displayed in the form of videos whose theme, content classification, display format and the length of time are different according to different focus points.

Training: classified video materials take event-group as directory and include the events of walking race, running, jump and throw. The events of walking race includes men and women race of different distances. Running includes sprints, middle and long distance running; jump includes 
high jump and long jump. Throw includes shot put throw, discus throw, javelin throw and weight throw. Material contents consist of miniature; medium and long videos edited with segments and whole set of technical movements, exercise load, psychological training, pre-event simulation, physical control of event training and demonstration.

Referee: this module includes track events referees (starting order, timer at the end, inspection and records, ranking at the end) and field events referees (throwing includes throwing shot put, discus, javelin, hammer; jump includes high jump, pole vault, long jump, triple jump etc.). The main theme is the video clips of referee's signs, cooperation, judgment scale of success, failure and the standards of site equipment., The software (Leawo video converter or Adobe Photoshop cc) is used to edit the videos and convert them to the format compatible with network players and store them in "My Resources" as an alternate material and they can be uploaded to video area for the reference of students.

$\mathrm{T} \& \mathrm{~F}$ events module is divided into events of primary and secondary schools, college T\&F events and international and national championships. Then materials of events organized by primary and secondary schools include the content, form, purpose and scale. College T\&F events includes the school, city, provincial, national and world events held by universities. It also includes T\&F combination forms, the technical characteristics, the psychological tactics athletes used to cope with the competition and the difference comparison between games of difference scales. The national and international championships include national indoor and outdoor T\&F championships and individual events competition. For international indoor and outdoor T\&F championships, the material are collected according to the technical characteristics, scale of referee rule, the comparison of characteristics of the record breaking athletes.

Classroom T\&F teaching includes teacher area and student area. Teacher area includes videos of the term's tasks, complete teaching organization process, presentation of teaching plan, teacher explanation and demonstration of events. Student area includes videos of warming up activities lead by students, presentation of teaching plan in groups and other videos of teaching.

Off campus teaching practice includes teaching support in poor areas, observation of teaching, teaching practice of training and competition. Teaching support is to help teaching in poverty-stricken areas, schools those have lack of resources. So, under the limited conditions, how to give a good T\&F lesson is a challenge. Its teaching model and characteristics are collected; observation of teaching means that physical education majoring students are regularly organized to observe some $\mathrm{T} \& \mathrm{~F}$ teaching models. Students can exchange ideas with the instructors on T\&F teaching in the field and the videos of summary session of the observation are collected.

$\mathrm{T} \& \mathrm{~F}$ research includes research methods, example and topic choosing guidance of bachelor degree thesis, T\&F academic subject classification and international research trends. Research methods mainly include lectures of themes, objects, methods, principles and analysis; thesis examples and thesis defense, and topic choosing guidance meeting; information collection of related $\mathrm{T} \& \mathrm{~F}$ academic conferences; lecture videos of international T\&F topic research trend.

Career guidance includes qualification certification, T\&F position and other employment issues. Certification means the relevant qualifications levels on T\&F as referees, coaches, exercise level, education achievement, which are recognized by society as a priority or condition when hiring employees. Athletics positions mainly refer to videos of recruitment scene of Sports Commission offices, sports schools, primary and secondary schools, primary training base which show the recruitment conditions and recruiting procedures. Employment issues are the employment problems under the current special circumstances, such as the required conditions of social physical education majors for teaching secondary schools, and lectures of how to obtain certifications.

$\mathrm{T} \& \mathrm{~F}$ health knowledge database include athletic injury, emergency protective measures and T\&F fitness philosophy. Athletic injuries include injuries got from T\&F teaching practice, training, organizing activities, different events and the corresponding injuries of different body parts. Emergency protective measures are taken to treat the injuries, such as the use of medical tools or postural placement and calling for help departments. T\&F fitness philosophy is video materials of adjustment activities after high-intensity exercise.

T\&F Celebrities' module includes Chinese celebrities and foreign celebrities in T\&F and experts on T\&F scientific research. $T \& F$ celebrities are athletes whose careers and outstanding achievements in T\&F at home and abroad are impressive and moving, and have an influence on the sports philosophy of the society. T\&F research experts are engaged in the research of T\&F sports, fitness and education. It can change the idea of athletes, the public, educators, coaches and administrator on T\&F and their application in practice, and which has practical significance.

\section{Teaching Tests Resource Management}

Self-Test Assignments (exercises): Self-test assignment resources module has assignment library and self-test assignment module management. Assignment library has the assignments of general course, elective courses and minor courses. The main contents of the general course include training principles, teaching principles, technical principles and referee rules. Elective courses include T\&F research, technical principles, training rules, teaching organization and fitness principle. Minor courses include T\&F skills, referee organization and competitions. Assignment compiled or assigned before are stored in libraries of various types (including assignment title, content, type and release time). Self-testing assignments are mainly used to compile assignments of the belonged grades, courses or groups (or classes).

Click "add assignment" to fill in the assignment title, select whether to display the assignment (yes, no), use integrated functions in the module (font, style, format, size, search, image, video, emoticons, color, mathematical symbols, line type, etc.) to fill in the brief introduction dialog box. The module displays select structure, the course (Track and Field Two --- Teaching Method) is in operation; enter assignment library through "select assignment" and the targeted assignments can be selected in the assignment list (Figure 4). 
The number of items displayed per page $(10,20,30$ items) can be adjusted in the assignment list of assignment library; input words into the assignment title dialog box to query assignment wanted, or query all assignments that are saved. The list displays assignment content, type, release time and operation (add), which enables further management of the assignment (Figure 4).

Self-Test (test papers): Test papers mainly test students' command level of the current taught of T\&F in general, elective and minor courses. There are eight sets of spare papers per semester and the papers are compiled according to the questions' difficulty level to help teachers arranging self-tests. The main contents of the general course include training principles, teaching principles, technical principles and referee rules. Elective courses include T\&F research, technical principles, training rules, teaching organization and fitness principle. Minor courses include T\&F skills, referee organization and competitions. Add papers: In the add paper module management, fill in the title of the paper and select papers.

Add from test paper library, users can set the number of items displayed per page $(10,20,30,50,75,100)$. Enter key words into paper title dialog box to query, or query in all items. Select in the paper list one of paper title, author, state, created time and enter the content input module, whether to display paper title (yes / no), whether to save the paper to library (yes / no), edit introduction (users can use multi-functional editor) and select structure (the course name --- T\&F Two, the specific location --- add new paper). Click "submit" to edit the paper, the module can set the question type (questions and answers, operational question, calculation question, gap filling question, true of false question, one-choice question, multiple choice, short answer question), add title, import question and return, and at the same time paper titles and descriptions are displayed (Figure 5).

Add essay questions: add question text (using the multifunction key) and then submit (or return) to enter the answer module setting (multi-function keys are available), graphics and videos of the answer, score and the correct answer can be set. In the property module, the difficulty (easy, medium, hard, very hard), score (can be designed as needed) and finish time can be (minutes) set by the users and then click submit (or return) to finish the operation (Figure 5).

Import questions: enter import question module, that is, get into all the question libraries of this on-line course. Choose questions types (all types, essay questions, questions and answers, operational question, calculation question, gap filling question, true or false question, onechoice question, multiple choice and short answer question). Users can choose any one of track and field course's column and click it to enter question library and select a certain question or questions through clicking the operation button to import the question or questions to paper module to perform paper compiling management (Figure 5).

\section{RESUlts AND ANALYSIS}

\section{A. My Resources Management Effect}

Figure 6 shows that $33.33 \%$ of the experts believe that the effect of resources management is very diversified, $53.34 \%$ experts believe it is diversified, and $13.33 \%$ be-

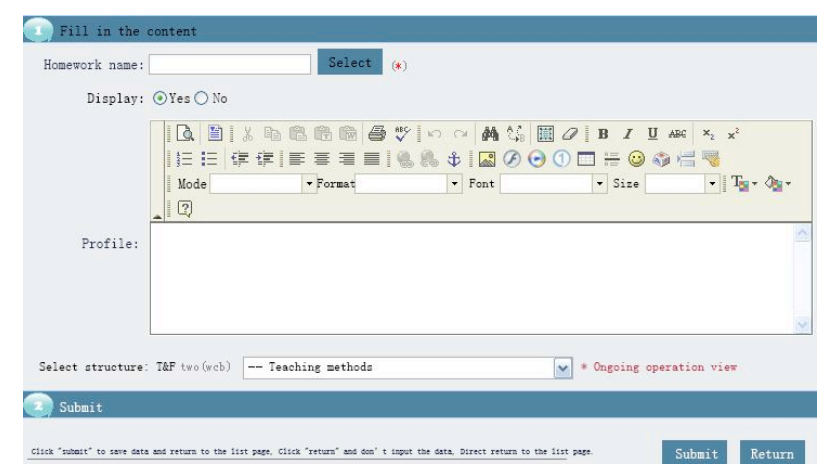

Figure 4. Add homework content

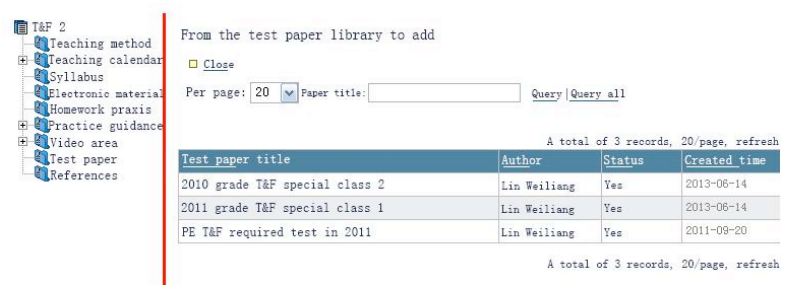

Figure 5. Add from test library

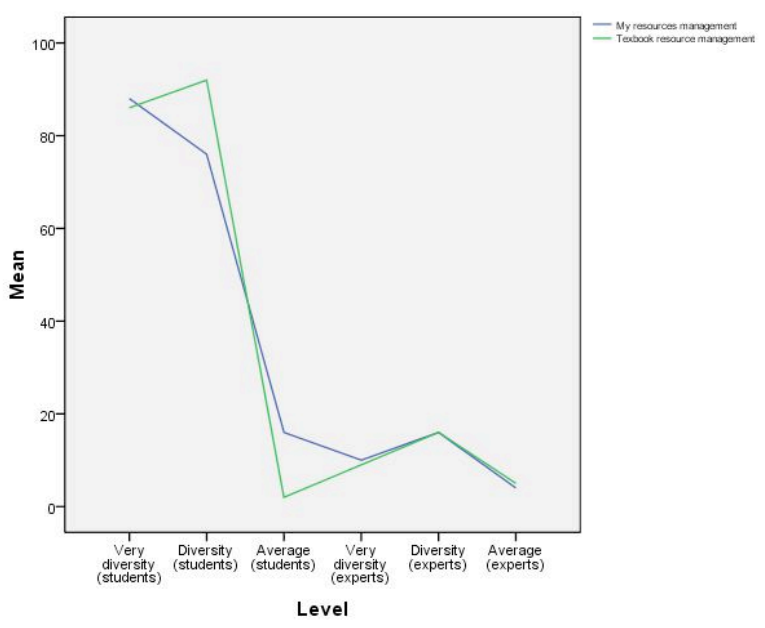

Figure 6. My resources and textbook management

lieves it is average. $48.89 \%$ of the students' evaluation is very diversified, $42.22 \%$ evaluated as diversified, and $8.89 \%$ average. None of them think it is monotonous. Generally, my resource module has a wealth of materials. Its contents are classified according to the requirements of the general list of teaching materials which includes the course syllabus, teaching structure, electronic textbooks, practice guidance, video area, assignments and exercises and self-tests.; Each module can be classified in multilevels, such as syllabus is divided into teaching methods, teaching calendar and syllabus. Course textbooks are divided into electronic materials (documents, books, articles), practical guidance $\mathrm{T} \& \mathrm{~F}$ training, referee, events, classroom teaching, teaching practice, scientific research, career guidance, health knowledge and track and field celebrities), videos area (consistent with the practice content), and testing resources are classified into self-teaching assignments and self-tests. Raw materials of My Resources are from documents issued by school and the edited existing syllabus teaching methodologies and teaching progress. Course books are from the latest pub- 
lished books, articles or open documents. Practice guidance are on-line resources extracted from representative cases of teachers, students, coaches, athletes and experts in the practice of teaching, refereeing, events, internship, research, job searching and keeping fitness. It is then edited by the Institute of Physical Education syllabus management of existing T\&F, teaching methods, teaching and progress to confirm. The curriculum materials are the latest published books, articles or public documents. Videos area is consistent with the practice content (the materials are recorded in videos, and miniature, middle and long videos are made according to its content characteristics to meet students' intuitive cognitive visual acceptance). The tests are from the assignments and test papers compiled and stored in assignment or test paper library by teachers (or administrator) according to the keys points of the course and the teaching progress. Storing a certain amount of assignments and self-testing papers during teaching period can keep the resources reserve adequate.

Multi-function keys are designed in My Resource module that can help perform comprehensive editing conveniently on the raw materials' title, content, files and text types, evaluation criteria (difficulty, score), graphics, text, video and color background with excellent visual effects. At the same time the transfer, editing and reuse of a teaching material corresponding to a certain resource can be carried out. Its modular material management has the feature of diversity and systematic characteristic.

\section{B. Teaching Structure Resource Management}

Figure 7 shows that $43.33 \%$ of the experts believe that the effect of the list level management is very clear, $30 \%$ believe it clear and $26.67 \%$ average. $50.56 \%$ of the students' evaluation is very clear, $37.22 \%$ evaluated as clear and $12.22 \%$ average. None of them thinks it is not clear. $50 \%$ of the experts believe that the effect of the structure adjustment is very clear, $36.67 \%$ clear, and $13.33 \%$ average. $58.33 \%$ of the students' evaluation is very clear, $40.56 \%$ evaluated as clear and $1.11 \%$ average. None of them thinks it is not clear. As a whole, teaching structure module can set its view names, edit content and open courses. Its directory contains materials of course syllabus, teaching structure, electronic textbooks, practice guidance, video area, assignments and exercises and selftests. Multifunction key can edit the names and texts of main directory and sub-directory. The directory order can be adjusted in each column: add directory, edit directory, locate, move and create similar. The contents added include directories such as files, texts, links, teaching reference, test papers, assignments, new test papers, my resources. Move: select one or more entries you want to transfer, click on the "transfer" button, select the directory you want to transfer from the pop-up page; the classification, induction and tree structure directory of teaching materials are conducive to the further examination, adjustment and guidance of the teaching structure.

\section{Teaching Syllabus Material Management Effect}

Figure 8 shows that $33.33 \%$ of the experts believe that the effect of the material representativeness management is very typical, $43.34 \%$ believe typical and $23.33 \%$ believe it as average. $61.67 \%$ of the students' evaluation is very

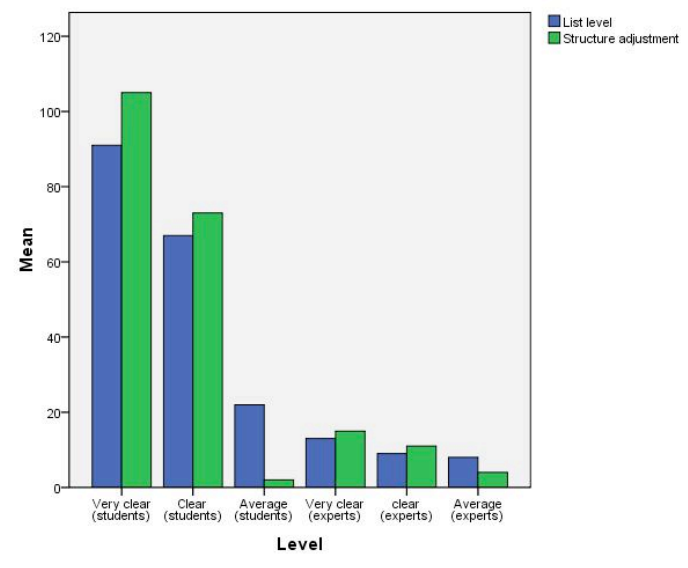

Figure 7. Teaching structure module

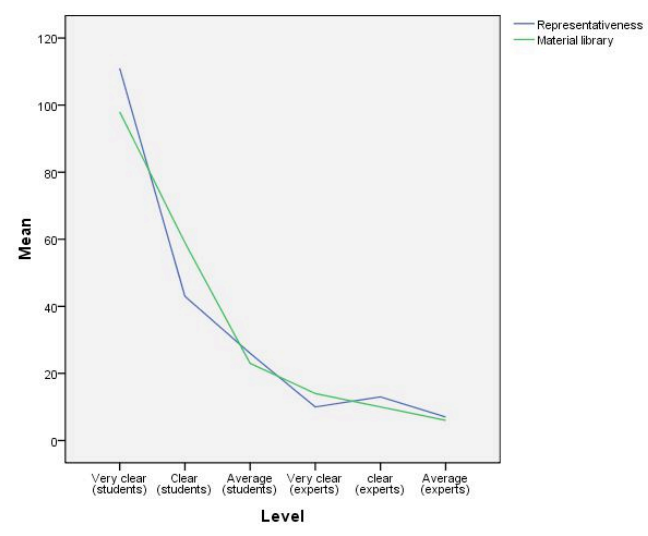

Figure 8. Teaching syllabus material management

typical, $23.89 \%$ evaluated as typical and $14.44 \%$ average. None of them thinks it is not typical. $46.67 \%$ of the experts believe that the effect of the material library adjustment is very typical, $33.33 \%$ think it is typical, $20.00 \%$ believe it is average. $54.44 \%$ of the students' evaluation is very typical, $32.78 \%$ evaluated as typical and $12.78 \%$ average. None of them thinks it is not typical. Generally, Syllabus includes teaching methods, teaching calendar and teaching guideline. It represents the general framework of the course. Teaching methods include the presenting form of the self-directed study of the current on-line courses, the mode of teaching activities and tasks finishing. The set of the management form of teaching evaluation module helps students to improve self-learning efficiency and help them get a quicker master of methods of studying the online courses. Teaching calendar in general teaching schedule made by the school before the semester begins. The timely collection of the relative information and the excel table of statistics are edited directly through the syllabus module and presented on the web course. The syllabus is subject to the latest revised syllabus whose subject is track and field compulsory courses, elective courses and minor courses. Its subdirectory set the location, background and the manifestation pattern of the resources like semester, teaching object, grades, course type etc. The course syllabus modules have professional editing effect on the resource setting. Its resources can be extracted from My Resources and the library can be reused and updated. 


\section{Textbook Resource Management Effect Analysis}

Figure 6 shows that $30 \%$ of the experts believe that the effect of the textbook resource management is very diversified, $53.33 \%$ believe it is diversified and $16.67 \%$ believe as average. $47.78 \%$ of the students' evaluation is very diversified, $51.11 \%$ evaluated as diversified and $1.11 \%$ evaluated average. None of them thinks it is monotonous. Generally, Course textbooks include electronic materials (documents, books, articles), practical guidance (T\&F training, referee, events, classroom teaching, teaching practice, scientific research, career guidance, health knowledge and T\&F celebrities), videos area (consistent with the practice content). Materials of each module are collected from real cases of different scenarios and prominent problems occurred in teaching. The software (Leawo video converter or Adobe Photoshop cc) is used to edit the raw video materials to control the quality and the length of the videos in order to meet the requirement of the web course module. Its practice themes and teaching tasks are consistent in theoretical system at some stage and its forward-looking features and innovative materials can better reflect the content topics. The module has a multifunctional, comprehensive network of setting the course material like voice-control video, image frames, background colors, rhythm playing and other factors can all be controlled by it. It help improve the visual effects of the resources and also convenient for teachers (administrator) to constantly update the resources.

\section{E. Teaching Tests Resource Management Analysis}

Figure 9 shows that $26.67 \%$ of the experts believe that the effect of the teaching tests resource management is very notable, $60.00 \%$ of them believe it is notable and $13.33 \%$ believe it as average. $43.33 \%$ of the students' evaluation is very notable, $55.00 \%$ of them evaluated it as notable, and $1.67 \%$ evaluated as average. None of them thinks that it is not notable. As a whole, teaching tests materials are mainly from existing assignment library, test paper library and question library; or they are compiled in real-time by editing the question types, titles, choices, answers, scores and difficulty levels in My Assignment module and self-test module. Its content reflects the requirement of T\&F teaching tasks, test the master level of the knowledge learned and indicate the inadequacy of the students. Its evaluation system has modular automatic feedback function and standard for evaluation. My Assignment has 16 questions of different stages of term, whose difficulty level are also different. Users can choose topic and difficulty level intently or randomly. Self-test module can refer to question and test paper library; teachers can edit and update questions and papers which is convenient for the compiling of test papers. 8 papers of different difficulty levels are prepared for every group or class according to the requirement of teaching objects, tasks and content for the students' self-test and selfevaluation. The orderly management of the materials in the teaching test module improves the systematic management effect of the module.

\section{F. Analysis of Resource Links Effect}

Figure 9 shows that $40.00 \%$ of the experts believe that the effect of the resource links is very notable, $50.00 \%$ of them believe that it is notable and $10.00 \%$ believe it as average; $58.89 \%$ of the students think that it is very notable, $26.67 \%$ think notable and $14.44 \%$ think it as average.

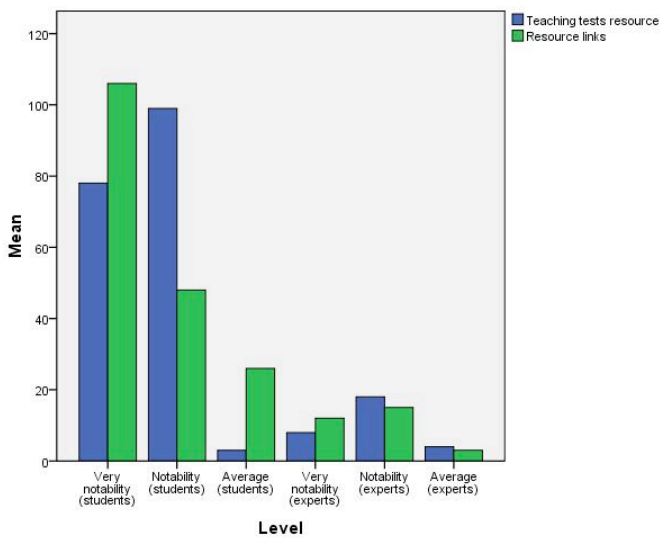

Figure 9. Teaching tests resource and resource links

None of them thinks it is not notable. As a whole, the interlinking effect between the module of my resources, teaching structure, syllabus, course textbooks and teaching tests is excellent. The link paths between the modules are clear and the actual operation is simple and is guided. These are conducive to the reference, moving, directory adjustment and automatic saving, and improve the system management effect. The modular dialog design of the course has functions of editing text, fonts, images, video, background color and images which help the administrator (teacher), perform comprehensive editing on different module resources, improve the visual effect and promote sustainable use of the resources.

\section{CONCLUSIONS}

T\&F web course teaching materials management includes my resources, teaching content and structure, course syllabus and course textbooks. My Resources contains teaching materials and users also constantly edit existing resources and compile new materials to update the module. The resource modules have essential characteristics, rich in content, interactive between modules and complementary with each other. It improves sharing and updating of the resources. The function of each module is diverse, convenient to operate and has the function of guidance that helps the editing, reference, and the efficiency of inter module linking. It is also with higher level of management mode of classification, guidance, add and save, which is conducive to the integration and organization of the implementation of track and field teaching tasks.

This research applies to physical education majors and the management of contents classification and multifunction setting of web course teaching materials resource. These are convenient for teachers and students to participate in this program to create resources together and help combine with T\&F practice teaching task. It is especially reflected in the $\mathrm{T} \& \mathrm{~F}$ web course under the current system reform condition in China.

\section{ACKNOWLEDGMENT}

This paper is one of the phased results of construction of track and field web course of Guangzhou University (Code: 0L1009322) and evaluation system of track and field web course of department of Physical Education (Code: G17). It is a 2010- present campus special research program funded by Guangzhou University and a 2015 
education evaluation project funded of Guangdong Assessment Association. Meanwhile, please allow me to express my gratitude to Guangzhou University. It was their help especially the T\&F teachers of school of physical education and sports science and others including the related students in research team who contributed significantly to conduct this research successfully.

\section{REFERENCES}

[1] Albert H. Huang, "A supply-chain management perspective of online education. Educational technology systems," vol. 29, no. .2, pp. 93-106, 2000.

[2] Brian W. Donavant, "The new, Modern practice of adult education: online instruction in a continuing professional education setting," Adult education quarterly, vol. 59, no..3, pp. 227-245, 2009, doll: $10.1177 / 0741713609331546$

[3] Brenda Dorcely, Nitin Agarwal, Maya Raghuwanshia, "Quality assessment of diabetes online patient education materials from academic institutions," Health Education Journal, vol.74, no.5, pp. 568-577, 2015, dol: 10.1177/0017896914552203.

[4] Cai Xin, "Network Vocational Training Design Based on Engagement Theory," China Audio Visual Education, vol. 6, no. 20, pp. 12-15, 2009 .

[5] Cathie Jensen-Lee, Annette Falahey, "Using the web: a discussion of some of the non-technical factors to be taken into account when designing web sites intended to support courses taught primarily in face-to-face mode," Journal of sociology, vol.38, no..3, pp.291301, 2002, dol: 10.1177/144078302128756679.

[6] Chen Li, Li Shuang, Ma Xiaoying, "Research on the Classification and demand of China's Distance Education Practitioners," China Distance Education, vol.11, no.1, pp.27-30, 2004.

[7] David John Dowell, Felicity A. Smal, "What is the impact of online resource materials on student self-learning strategies?", Journal of marketing education, vol.33, no.2, pp.140-148, 2011, doll: 10.1177/0273475311410846.

[8] DI Xu, “Across Virginia' s community colleges: evidence from introductory college-level Math and English courses," Evaluation and policy analysis, vol. 33, no.3, pp.360-377, 2011.

[9] Fang Xiaodong, "System of educational resource management for vocational education based on industrial culture," China Distance Education, vol.15, no.1, pp.61-63, 2013.

[10] J.B. Arbaugh, "What might online delivery teach us about blended management education? Prior perspectives and future directions," Journal of management education, vol.38, no. 6, pp.784-817, 2014, dol:10.1177/1052562914534244.

[11] Jessica M. Lee, MD,Forrest Farnandez MD, FACS, Ilene Staff, $\mathrm{PhD}$, John W. Mah, MD, FACS, “Web-based teaching module improves success rates of Postpyloric Positioning of nasoenteric feeding tubes," Journal of Parenternal \& Enteral nutrition, vol.36, no..3, pp.323-329, 2012, dol. 10.1177/0148607111416246.

[12] Lalla N Boisselle, "Online-learning and its utility to higher education in the Anglophoe Caribbea," Sage open, vol.4, no.4, 2014 , dol.2158244014555118.

[13] LiJun, Yu Ping, "Analysis and Functional module of the management system of Net -Course," Journal of Jingdezhen College, vol.21, no.2, pp.41-42, 2006.
[14] Liu Tao, Wang Suli, Feng Jianwen, Zeng Qiao Lian, "The construction of network course management system based on basic computer course," Software Guide, vol. 9, no. 5, 142-143, 2010.

[15] Luo Shaoke, Sun Zhongjin, "Research on Video Courses Resources Management of Open Network Course," Popular Science \& Technology, vol. 4, no.4, pp.159-160, 2009.

[16] Martin Forsey, Mitchell Low, David Glance, "Flipping the sociology classroom: Toward a practice of online pedagogy," Journal of Sociology, vol.49, no.4, pp.471-485, 2013, dol: 10.11771440783313504059 .

[17] Nicholas C. Burbules, “ Aporlas, Webs, and passages: Doubt as an opportunity to learn," Curriculum inquiry. vol. 30, no. 2, pp.171187, 2000, Dol: 10.1111/0362-6784.00161.

[18] Peter Williams, "The learning web the development, implementation and evaluation of internet-based undergraduate materials for the teaching of Key Skills," Active learning in higher education, vol.3, no.1, pp.40-53, 2002, doll: 10.1177/1469787402003001004.

[19] Richard W. Willson, "In-class-online hybrid methods of teaching planning theory- assessing impacts on discussion and learning," Journal of planning education and research, vol. 28, no.2, pp.237248, 2008, dol: 10.1177/0739456x08324286.

[20] Ruan Guo-long, Liu Feng, "Discussion on the mode of managing web-based course based on material," Journal of Xianning College, vol. 24, no. 6, pp.48-53, 2004.

[21] Weiliang Lin, "Research on the design mode of web course of track \& field in physical education in college," International Journal of Emerging Technologies in Learning, vol.9, no.3, pp.68-72, 2014. http://dx.doi.org/10.3991/ijet.v9i3.3285

[22] Weiliang Lin, "Research on university track and field web course's basic attributes columns," International Journal of Emerging Technologies in Learning, vol.4, no.8, pp.27-33, 2013. http://dx.doi.org/10.3991/ijet.v8i4.2941

[23] Weiliang Lin, "Track and Field online curricula evaluation system design for universities physical education," International Journal of Emerging Technologies in Learning, vol.4, no. 8, pp.10-16, 2013. http://dx.doi.org/10.3991/ijet.v8i4.2940

[24] Weiliang Lin, "Research on The Model of Test Module from Track and Field Wed Course to Physical Education in College," 2013 Fourth international conference on education and sports education, vol.11, no.14, pp.347-354.

[25] Wu-Yuin Hwang, Shih-ching Yeh, Stephen J.H.Yang, Jing-Liang Wang, Lin Han, Guo-Liang Hsu, "Effects of unidirectional vs. reciprocal teaching strategies on web-based computer programming learning," Journal of educational computing research, vol.50, no.1, pp. 67-95, 2014. dol. 10.2190/EC.50.1.d.

[26] Zhang Yin, "Analysis and Thinking on the Mechanism of Answering System," China Distance Education, vol.12, no.2, pp. 23-25, 2006.

\section{AUTHOR}

Weiliang Lin is working with the School of Physical Education and Sports Science of Guangzhou University, Guangzhou, China (e-mail: linweiliang70@163.com).

This work is a 2010- present campus special research program funded by Guangzhou University and a 2015 education evaluation project funded by Guangdong Assessment Association.

Submitted 12 January 2016. Published as resubmitted by the author 09 March 2016. 